\title{
Highly efficient derivation of ventricular cardiomyocytes from induced pluripotent stem cells with a distinct epigenetic signature
}

Huansheng $\mathrm{Xu}^{1,2,3,{ }^{*}}$, B Alexander $\mathrm{Yi}^{1,2,3,{ }^{*}}$, Hao $\mathrm{Wu}^{1,2,3}$, Christoph Bock ${ }^{2,3,4}$, Hongcang $\mathrm{Gu}^{2,3,4}$, Kathy O Lui ${ }^{1,2,3}$, Joo-Hye C Park ${ }^{1}$, Ying Shao ${ }^{1}$, Alyssa K Riley ${ }^{1}$, Ibrahim J Domian ${ }^{1,2}$, Erding Hu ${ }^{5}$, Robert Willette ${ }^{5}$, John Lepore ${ }^{5}$, Alexander Meissner ${ }^{2,3,4}$, Zhong Wang ${ }^{1,2}$, Kenneth R Chien ${ }^{1,2,3}$

${ }^{I}$ Cardiovascular Research Center, Massachusetts General Hospital, Charles River Plaza/CPZN 3208, 185 Cambridge Street, Boston, MA 02114, USA; ${ }^{2}$ Harvard Stem Cell Institute; ${ }^{3}$ Department of Stem Cell and Regenerative Biology, Harvard University, 7 Divinity Avenue, Cambridge, MA 02138, USA; ${ }^{4}$ Broad Institute, Cambridge, MA 02142, USA; ${ }^{5}$ Cardiac Biology, HF Discovery Performance Unit, Metabolic and Cardiovascular Therapeutic Area Unit, GlaxoSmithKline Pharmaceuticals, 709 Swedeland Road, King of Prussia, PA 19406, USA

Cardiomyocytes derived from pluripotent stem cells can be applied in drug testing, disease modeling and cellbased therapy. However, without procardiogenic growth factors, the efficiency of cardiomyogenesis from pluripotent stem cells is usually low and the resulting cardiomyocyte population is heterogeneous. Here, we demonstrate that induced pluripotent stem cells (iPSCs) can be derived from murine ventricular myocytes (VMs), and consistent with other reports of iPSCs derived from various somatic cell types, VM-derived iPSCs (ViPSCs) exhibit a markedly higher propensity to spontaneously differentiate into beating cardiomyocytes as compared to genetically matched embryonic stem cells (ESCs) or iPSCs derived from tail-tip fibroblasts. Strikingly, the majority of ViPSC-derived cardiomyocytes display a ventricular phenotype. The enhanced ventricular myogenesis in ViPSCs is mediated via increased numbers of cardiovascular progenitors at early stages of differentiation. In order to investigate the mechanism of enhanced ventricular myogenesis from ViPSCs, we performed global gene expression and DNA methylation analysis, which revealed a distinct epigenetic signature that may be involved in specifying the VM fate in pluripotent stem cells.

Keywords: induced pluripotent stem cells; ventricular cardiomyocyte; regenerative medicine; epigenetic memory Cell Research (2012) 22:142-154. doi:10.1038/cr.2011.171; published online 8 November 2011

\section{Introduction}

Embryonic stem cells (ESCs) and induced pluripotent stem cells (iPSCs) [1], as well as the direct reprogramming of fibroblasts into cardiomyocytes $[2,3]$, are potential sources of cardiomyocytes for drug testing, disease modeling and cell-based therapy. However, a number of critical factors need to be addressed before these innovations can be translated practically. For instance, can they

*These two authors contributed equally to this work.

Correspondence: Kenneth R Chien

E-mail: krchien@partners.org

Received 16 September 2011; revised 28 September 2011; accepted 29 September 2011; published online 8 November 2011 efficiently produce adequate numbers of functionally beating cardiomyocytes for clinical testing? It is estimated that a billion myocytes are lost after a myocardial infarction [4], and while pluripotent stem cells have been used to produce cardiomyocytes for small-scale in vivo animal studies, the production of cardiomyocytes from pluripotent stem cells or by direct reprogramming methods is generally inefficient and the yields are typically low $[2,3,5,6]$. Neither pluripotent stem cells nor direct reprogramming methods address another critical issue, which is the need to generate chamber-specific cardiomyocytes. Pacemaker, atrial, and ventricular myocytes (VMs) have distinct functional and electrophysiological properties that may contribute to cardiac arrhythmias in the wrong environment $[7,8]$. Pluripotent stem cells 
yield a heterogeneous population of cardiomyocytes of which only $30 \%-70 \%$ are VMs $[9,10]$, and methods of purifying VMs from a population of stem cell-derived cardiomyocytes remain to be established.

These issues underscore the need to better understand the molecular pathways that govern the specification of VMs from pluripotent stem cells. It has been demonstrated that iPSCs derived from different somatic cell types retain an epigenetic memory of the starting cell type that confers a tendency to redifferentiate back to their parental cell types [11-15]. These results prompted us to consider whether ventricular cardiomyocyte-derived iPSCs might serve as a source of VMs. Here, we report the derivation and characterization of iPSCs from VMs (ViPSCs). ViPSCs exhibited a dramatically increased propensity to form cardiovascular progenitors and differentiate into functionally beating cardiomyocytes. Interestingly, stem cell "memory" in ViPSCs also directs differentiation towards the VM phenotype. Global gene expression and DNA methylation analysis of ViPSCs reveal a unique transcriptional and epigenetic signature that likely plays a key role in ventricular myogenesis from pluripotent stem cells. The ability to derive large numbers of chamber-specific VMs from pluripotent stem cells would address critical issues in the advancement of cardiomyocyte replacement therapy for heart disease.

\section{Results}

\section{Generation of iPSCs from VMs}

In order to eliminate the potentially confounding effects of varying integration sites of the reprogramming transgenes, we generated genetically matched iPSCs starting from VMs and tail-tip fibroblasts (TTFs) using a conditional reprogramming system (Figure 1A) [16, 17]. We isolated cardiac fibroblasts from neonatal pups with the compound genotype $M l c 2 v^{C r e /+} ; \operatorname{Ros} a 26^{A 0 x Y F P / r T T A}$; TgMef2c-AHF-dsRed. The Mlc2v $v^{\mathrm{Cre}}$ knock-in allele [18] and a Cre-dependent conditional $\operatorname{Rosa} 26^{f l o x Y F P}$ reporter allele (with a loxP-flanked stop signal before the yellow fluorescent protein (YFP) cDNA) [19] specifically and permanently label ventricular cardiomyocytes with YFP (Figure 1B). The Rosa26 $6^{r T A}$ knock-in allele constitutively expresses rtTA, which encodes the transactivator for the doxycycline-inducible promoter [20]. Finally, the Mef2c$A H F$-dsRed transgene labels Islet- $1^{+}$cardiovascular progenitors of the anterior heart field (AHF) and their descendants (primarily the right ventricle and cardiac outflow tract) with dsRed (Figure 1B) [21, 22].

We derived primary iPSC clones by transducing the cardiac fibroblasts with lentiviruses that express the reprogramming factors Klf4, Oct3/4, Sox2, and Myc under the control of a doxycycline-inducible promoter. After injection into wild-type blastocysts, the primary iPSC clones rendered chimeric postnatal pups. Analysis of chimeras revealed that iPSC derivatives were $\mathrm{YFP}^{+}$in the cardiac ventricles and dsRed ${ }^{+}$in AHF-derived structures, indicating that the double reporter system retained their activity upon reprogramming to iPSCs (Figure 1C). From a single chimeric postnatal pup, YFP-positive VMs as well as TTFs (YFP-negative; Figure 1D) were isolated and replated on a mouse embryonic fibroblast (MEF) feeder cell layer under ESC conditions with doxycycline to generate secondary iPSCs from VM and TTF. Within 2 weeks, cells with an ESC-like morphology emerged (Figure 1E).

Colonies that maintained an ESC-like morphology in the absence of doxycycline were picked and expanded. Gene expression analysis demonstrated that ESC-like colonies from both VM (ViPSC) and TTF (TiPSC) expressed levels of Nanog, Oct3/4 (Pou5f1), and Sox2 that were comparable to mouse ESCs (Supplementary information, Figure S1A). These colonies displayed alkaline phosphatase activity and also demonstrated the expression of SSEA1, Nanog, and Oct4 by immunofluorescence (Supplementary information, Figure S1B and S1C). When grafted under the kidney capsules of the NOD/SCID immunodeficient mice, ESC-like colonies from both VM and TTF differentiated into teratomas containing all three germ layers (Supplementary information, Figure S1D). Of the multiple lines of ViPSCs and TiPSCs with the correct karyotype, six of each were randomly selected for further analysis as well as six de novo ESC lines from transgenic blastocysts with the $\mathrm{Ml}$ $c 2 v^{\text {Crel+ }} ;$ Rosa $26^{\text {foxYFP/rtTA }} ;$ TgMef $2 c-A H F-d s R e d$ genotype.

ViPSCs have a tendency to contribute to the heart upon blastocyst injection in vivo

We examined the developmental potential of ViPSCs, TiPSCs, and ESCs in vivo by injecting them into $2 \mathrm{n}$ blastocysts for chimera formation and examined embryos at embryonic day 13.5 (E13.5). While ViPSCs were already $\mathrm{YFP}^{+}$, TiPSCs and ESCs were $\mathrm{YFP}^{-}$and not readily visible. To visualize the descendents of injected TiPSCs and ESCs in the chimeras, we irreversibly activated the expression of YFP from the Rosa $26^{\text {AoxYFP }}$ allele by transient expression of an exogenous Cre in the injected cells. Chimeric embryos were obtained from all tested ViPSC lines and TiPSC lines. Interestingly, we observed that the pattern of ESC/iPSC contribution to the chimeras could be categorized into three types (Figure 2A and 2B): type 1 chimeras demonstrated a relatively broad and even pattern of chimeric contribution; type 2 chimeras demonstrated a disproportionally high contribution to the heart; 


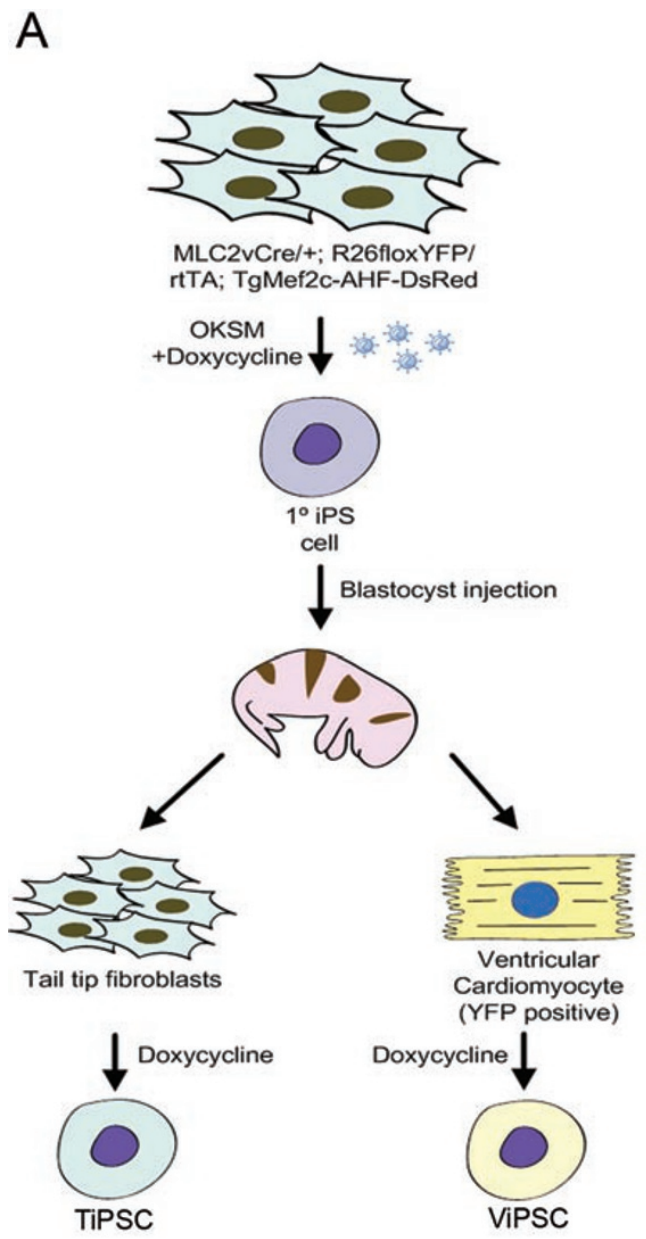

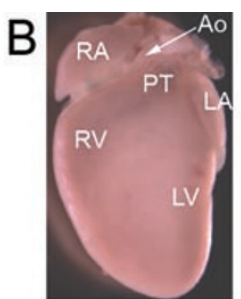
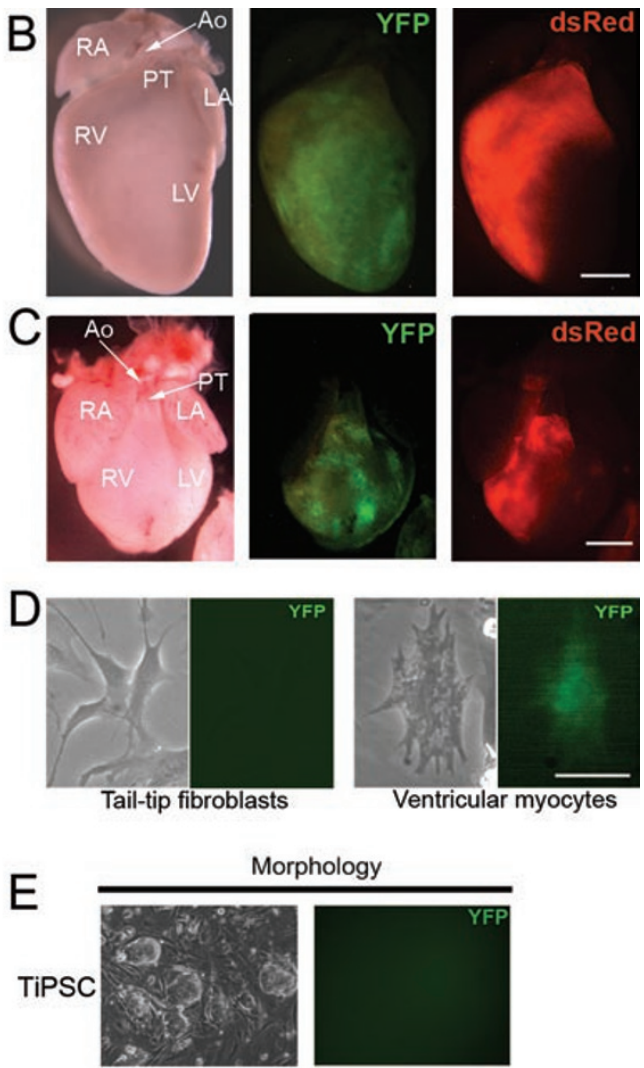

Morphology

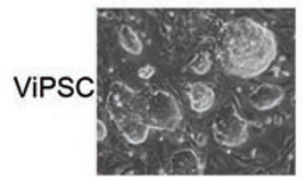

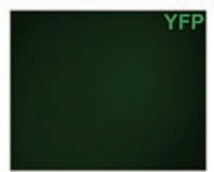

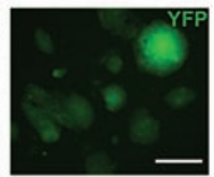

Figure 1 Generation of secondary mouse iPSCs. (A) The strategy to generate iPSCs from murine ventricular cardiomyocytes

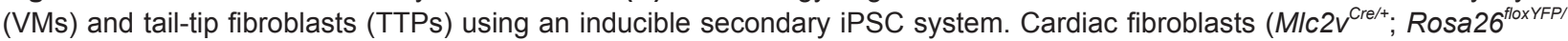
ITA; TgMef2c-AHF-dsRed) were isolated and transduced with separate lentiviruses containing doxycycline-inducible reprogramming factors, Oct4, Sox2, Klf4, and Myc, in order to derive iPSC clones. Primary iPSCs were injected into blastocysts to generate chimeras. TTFs and VMs were isolated from a single postnatal day 0 (P0) chimeric pup. After isolation, the cells were seeded onto an irradiated MEF feeder cell layer and doxycycline was applied for 2 weeks. Secondary iPSCs were picked on the basis of ESC-like colony morphology and doxycycline-independent growth. (B) Whole mount fluorescence microscopy of a P3 heart (left panel, bright field) from Mlc $2 v^{\text {Cre/t+}} ;$ Rosa26 floxYFP/ITA $;$ TgMef2c-AHF-dsRed compound transgenic mouse. Both ventricles (RV and LV) are labeled by YFP (middle panel) whereas the right ventricle (RV), pulmonary trunk (PT), and ascending aorta (Ao) are labeled by dsRed (right panel). RA and LA: right atrium and left atrium. Scale bar, $500 \mu \mathrm{m}$. (C) Whole mount fluorescence microscopy of the heart (left panel, bright field) from a P0 chimeric animal that was derived by blastocyst injection of primary iPSCs. After factor-based reprogramming and blastocyst injection, the double reporter system remains active with iPSCs that had contributed to both ventricles (RV and LV) being labeled with YFP (middle panel) and iPSCs that had contributed to the right ventricle (RV), pulmonary trunk (PT), and ascending aorta (Ao) labeled by dsRed (right panel). RA and LA: right atrium and left atrium. Scale bar, $500 \mu \mathrm{m}$. (D) Bright field and fluorescent images of TTPs and VMs isolated from the chimeric pup. Note that TTFs were YFP', while VMs were $\mathrm{YFP}^{+}$due to expression of Mlc2 $v^{\mathrm{Cre}}$ in the chimera. Scale bar, $20 \mu \mathrm{m}$. (E) ESC-like colonies that emerged upon seeding TTFs and VMs onto irradiated MEFs in ESC medium with doxycycline. Colonies derived from VMs retain expression of YFP. Scale bar, $200 \mu \mathrm{m}$.

and type 3 embryos demonstrated a relatively poor chimeric contribution in general. In contrast to the chimeras from ESCs (3 out of 12, or 25\%) and TiPSCs ( 0 out of $24)$, more than half of the chimeras (10 out of $19,53 \%)$ from ViPSCs were of type 2, in which the contribution of the iPSCs were concentrated in the heart. The distribution pattern of the various chimera types is significantly different between the chimeras from ViPSCs and those 
from ESCs or TiPSCs $(P=0.038$ between ViPSCs and ESCs, $P=0.002$ between ViPSCs and TiPSCs, $\chi^{2}$-test). This result suggests that ViPSCs have a predisposition to differentiate into cardiac lineages in the developing embryo.
A
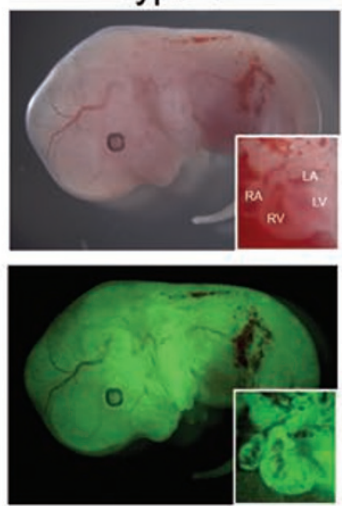

B

\begin{tabular}{ccccc}
\hline & Type 1 & Type 2 & Type 3 & Total \\
\hline ESC & $4(33 \%)$ & $3(25 \%)$ & $5(42 \%)$ & $12(100 \%)$ \\
\hline ViPSC & $8(42 \%)$ & $10(53 \%)$ & $1(5 \%)$ & $19(100 \%)$ \\
\hline TiPSC & $18(75 \%)$ & $0(0 \%)$ & $6(25 \%)$ & $24(100 \%)$ \\
\hline
\end{tabular}

C
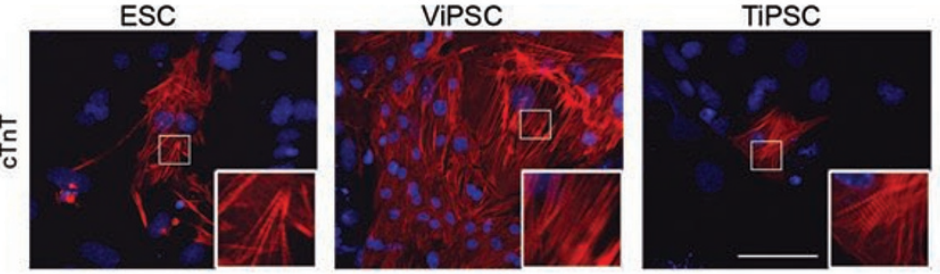

D
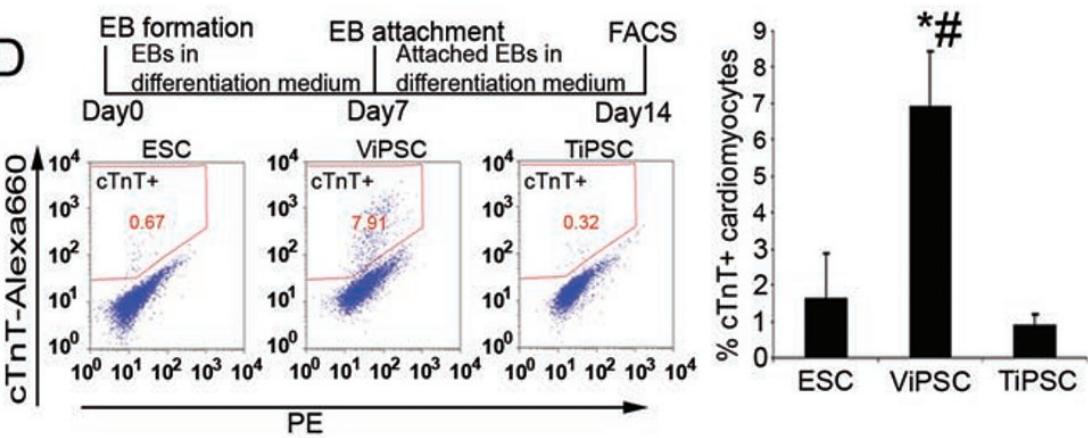

E

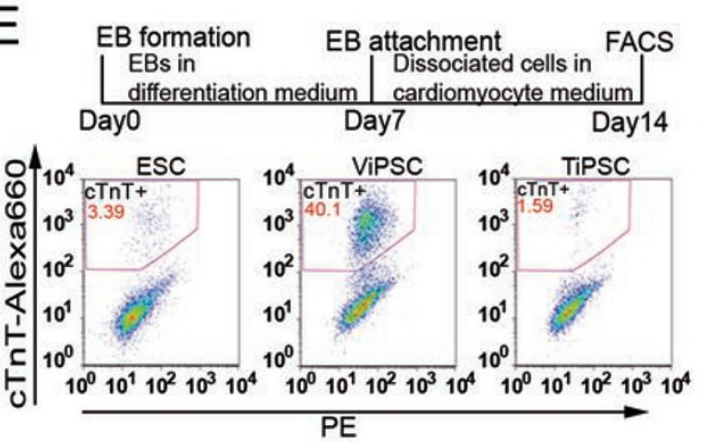

Type 3
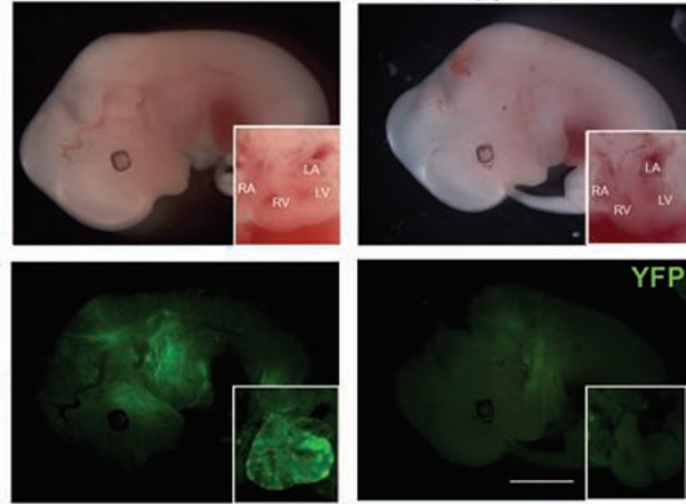

YFP

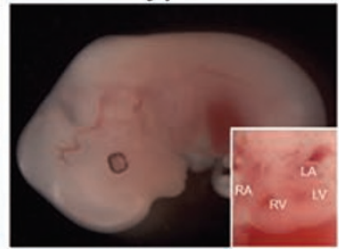

(1)

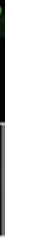


ViPSCs spontaneously redifferentiate into cardiomyocytes in an efficient manner in vitro

In order to compare their differentiation potential in vitro, we differentiated ViPSCs, TiPSCs as well as ESCs (all lines at passage 8) by embryoid body (EB) formation. ViPSCs, TiPSC, and ESC formed EBs of comparable size (data not shown). While the EBs derived from ESCs and TiPSCs did not begin contracting until $\sim$ EB day 8 , strikingly, spontaneously contracting EBs from ViPSCs were observed by day 6 . On day 8 , about $72 \%$ of EBs from ViPSCs demonstrated spontaneous contraction. After plating onto gelatin-coated dishes, the number of spontaneously beating EBs were monitored daily. At all examined time points, the overall percentage of contracting EBs from ViPSCs were higher than TiPSC or ESC (Supplementary information, Figure S2A). This was also consistent when monitoring expression levels of the cardiomyocyte marker, cardiac Troponin T (cTnT), along the time course of EB differentiation (Supplementary information, Figure S2B). By immunofluorescence, cells from day 14 EBs stained positive for $\mathrm{cTnT}$ in a sarcomere-like pattern (Figure 2C).

We quantified the yield of cardiomyocytes by performing intracellular fluorescence-activated cell sorting (FACS) using an antibody against cTnT. On day 14, ViPSCs yielded a significantly higher percentage of $\mathrm{cTnT}^{+}$ cells $(6.9 \%$, average of six lines) than ESCs $(1.7 \%$, average of six lines) or TiPSCs $(0.9 \%$, average of six lines $)$ (Figure 2D). Furthermore, higher expression levels of cardiogenic genes were detected in EBs from ViPSCs (Supplementary information, Figure S2C). EB aggregates may not be optimal for the generation and maintenance of large numbers of beating cardiomyocytes [23]; thus, we dissociated day 7 EBs into individual cells and then cultured them for another week to simulate monolayer culture conditions. With this protocol, ViPSCs yielded $\sim 30-40 \%$ cardiomyocytes - an increase of more than 10-fold compared to TiPSCs or ESCs (Figure 2E). These results suggest that cardiomyocyte-derived iPSCs retain memory of their starting cell type.

\section{ViPSCs are highly ventriculomyogenic}

ESCs and iPSCs differentiate into a heterogeneous mixture of atrial, nodal, and ventricular cardiomyocytes that display distinct functional and electrophysiological properties [9]. Since ViPSCs were derived from VMs, we wondered whether ViPSCs also had a predilection for differentiating specifically into VMs. We performed double immunostaining on differentiated ViPSCs, TiPSCs, and ESCs with a cTnI antibody that recognizes both atrial myocytes and VMs and an Mlc2v antibody that specifically recognizes VMs (Figure 3A). In all, $92 \%$ (average of six lines, with four lines $>90 \%$ and the maximum $99.5 \%$ ) of the $\mathrm{cTnI}^{+}$cardiomyocytes derived from ViPSCs also stained with the VM marker Mlc2v, while the percentage of cardiomyocytes that stained with Mlc2v was significantly lower for ESCs $(59 \%$, average of six lines) and TiPSCs (62\%, average of six lines; Figure 3B). Furthermore, gene expression analysis performed on the FACS-purified $\mathrm{cTnT}^{+}$fraction from ViPSCs demonstrated a $55 \%$ higher Mlc2v/cTnT ratio of gene expression than the $\mathrm{cTnT}^{+}$fractions from TiPSCs or ESCs (Figure 3C). Finally, patch clamp recordings demonstrated that $94 \%$ (15 out of 16 recordings) of ac-

Figure 2 ViPSCs display increased cardiomyogenic potential in vivo and in vitro. (A) Images of E13.5 embryos obtained by injecting ESCs, ViPSCs, or TiPSCs into $2 n$ blastocysts. ViPSCs were already YFP ${ }^{+}$upon derivation and readily visible in the chimeras, while ESCs and TiPSCs were not. To visualize the descendents of injected ESCs and TiPSCs in the chimeras, YFP from the Rosa26 floxYFP allele was irreversibly activated by the transient expression of an exogenous Cre. The pattern of ESC/iPSC contribution to the chimeras could be classified into three types: type 1 chimeras demonstrated an overall $\geq 50 \%$ contribution to the entire chimera and a similar contribution to the heart; type 2 chimeras demonstrated a $<50 \%$ contribution overall but a much higher contribution to the heart; and type 3 embryos that demonstrated a $<50 \%$ contribution overall with an equal or lesser contribution to the heart. Scale bar, $2 \mathrm{~mm}$. (B) Tabulation of the patterns of contribution of ViPSCs, TiPSCs, and ESCs to mouse chimeras in A. More than half of ViPSCs displayed a restricted contribution to the chimeric heart a pattern of contribution that was not observed with TiPSCs. (C) CTnT immunostaining of cardiomyocytes derived from ESCs, ViPSCs, and TiPSCs. Nuclei were stained with DAPI. Insets show the striated pattern of cTnT (insets). Scale bar, $10 \mu \mathrm{m}$. (D) Cardiomyogenic potential of ViPSCs compared to ESCs and TiPSCs, as revealed by FACS analysis for $\mathrm{CTnT}^{+}$cells after differentiation by EB formation and cultivation of whole EBs. Left, representative FACS dotplots with cTnT ${ }^{+}$cell percents. Right, bar graph showing statistical analysis of six lines of ESC, ViPSC, and TiPSC. Asterisk denotes significantly different from ESC lines, $P<0.05$; pound sign denotes significantly different from TiPSC lines, $P<0.05$, $t$-test. Error bars represent standard error. (E) Cardiomyogenic potential of ViPSCs compared to ESCs and TiPSCs, as revealed by FACS analysis for cTnT ${ }^{+}$ cells after differentiation by EB formation and cultivation of dissociated cells. Left, representative FACS dotplots with cTnT ${ }^{+}$ cell percents. Right, bar graph showing statistical analysis of six lines of ESC, ViPSC, and TiPSC. Asterisk denotes significantly different from ESC lines, $P<0.05$; pound sign denotes significantly different from TiPSC lines, $P<0.05$. $n=6$ lines for each cell type, $t$-test. Error bars represent standard error. 
tion potentials recorded from the beating cells differentiated from six ViPSC lines were of ventricular type with a rapid upstroke, distinct plateau phase, and stable resting membrane potential (Figure 3D) [24-26]. Furthermore, action potentials of ventricular type were also prevalent when it came to individual ViPSC lines, with cardio-
A

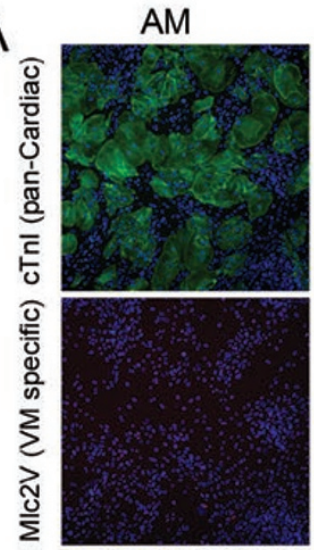

VM

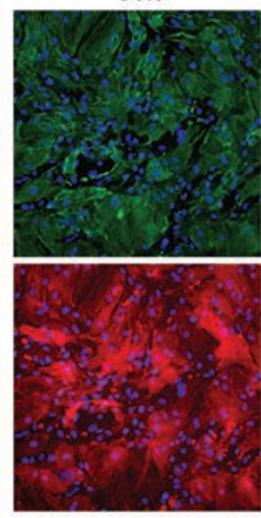

ESC
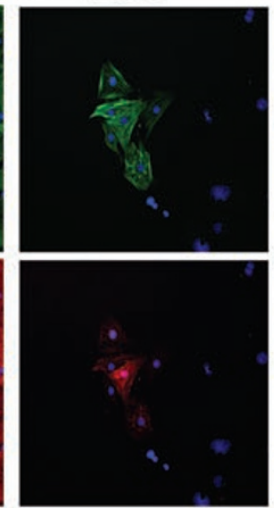

ViPSC
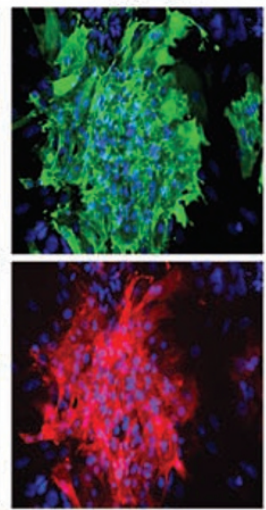

TiPSC

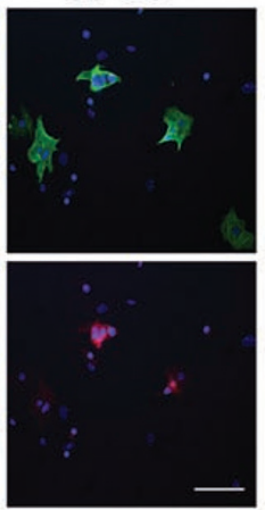

B

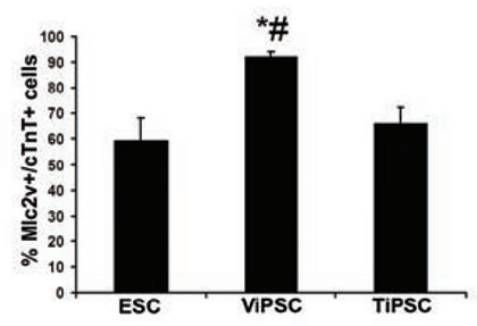

C
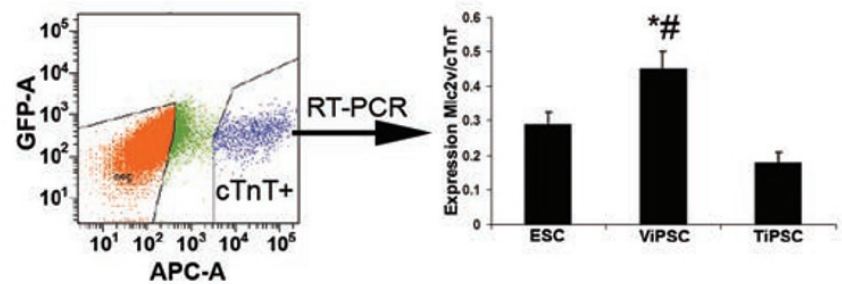

APC-A

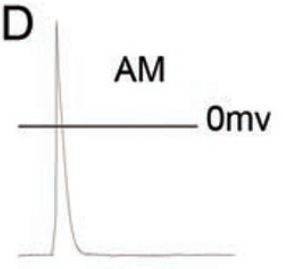

E

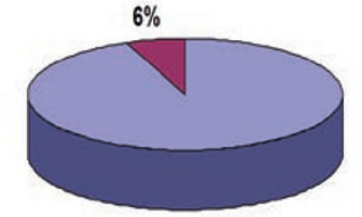

$94 \%$

口Ventricular type AP $\square$ Non-ventricular type AP
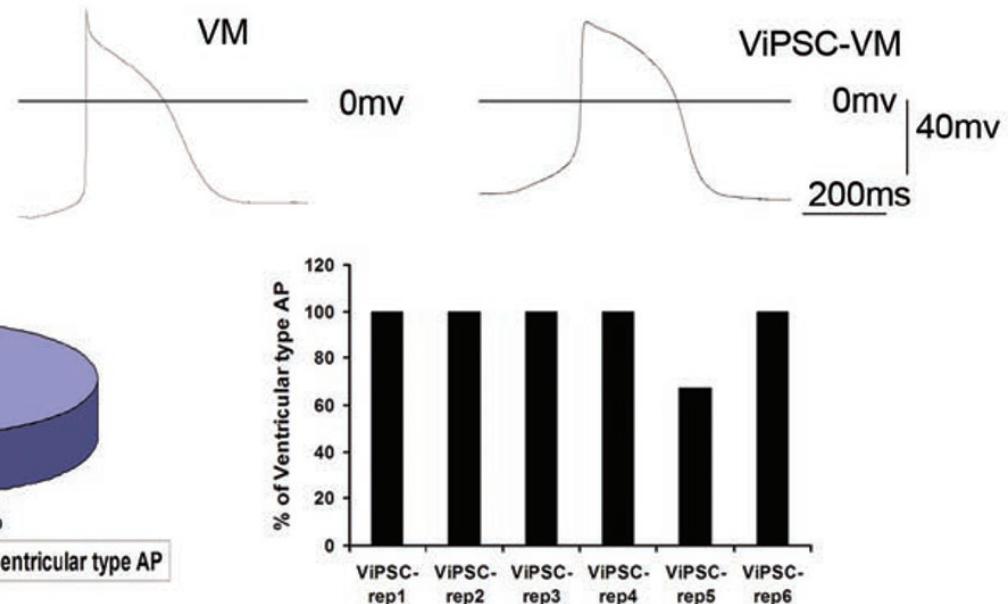

Figure 3 Cardiomyocytes derived from ViPSCs display a ventricular phenotype. (A) Double immunostaining for cTnl and Mlc2v from neonatal atrial myocytes (AM), ventricular myocytes (VM), and cells derived from ESCs, ViPSCs, and TiPSCs. Scale bar, $10 \mu \mathrm{m}$. (B) Bar graph showing percentage of ventricular myocytes $\left(\mathrm{Mlc}^{2} \mathrm{v}^{+}\right)$among all cardiomyocytes $\left(\mathrm{cTnl} \mathrm{I}^{+}\right)$ derived. Asterisk denotes significantly different from ESC lines ( $n=6$ cell lines), $P<0.05$; pound sign denotes significantly different from TiPSC lines ( $n=6$ cell lines), $P<0.05 . n=6$ cell lines for ViPSCs. Bars represent standard error. (C) Gene expression analysis of the FACS-purified $\mathrm{CTnT}^{+}$fraction. Asterisk denotes significantly different from ESC lines ( $n=6$ cell lines), $P<0.05$; pound sign denotes significantly different from TiPSC lines ( $n=6$ cell lines), $P<0.05 . n=6$ cell lines for ViPSCs. Bars represent standard error. (D) Representative action potential recorded from a spontaneously contracting atrial myocyte (AM), ventricular myocyte (VM), and a ViPSC-derived ventricular myocyte (ViPSC-VM). (E) Overall distribution of recorded action potentials from ViPSC-derived cardiomyocytes (left, pie chart, 16 recordings) and the distribution of action potentials for each ViPSC line (right, bar chart). 
myocytes from $83 \%$ (five out of six lines) of the lines displaying action potentials exclusively of ventricular type (Figure 3E). These data suggest that "memory" in ViPSCs extends to the VM lineage.

Enhanced generation of cardiovascular progenitors in ViPSCs

Cardiomyocytes are derived from multipotent cardiovascular progenitors, such as those marked by the expression of Islet-1 or $N k x 2.5$, that become progressively lineage restricted before terminally differentiating to the cardiomyocyte fate $[22,27,28]$. To gain insight into the cellular pathway by which ViPSCs differentiate into VMs, we analyzed EBs at early stages for Mef2c-AHF$d s R e d$ positive cells by FACS analysis. EBs at day 7 displayed a higher number ( $>10$-fold) of $\mathrm{dsRed}^{+}$cells in ViPSCs EBs than TiPSCs EBs (Figure 4A and 4B). Furthermore, gene expression of early stage EBs revealed higher levels of $N k \times 2.5$ and Islet-1 expression in ViPSCs than TiPSCs or ESCs (Figure 4C), suggesting that upon differentiation, ViPSCs form cardiovascular progenitors at higher efficiency than conventional iPSCs or ESCs and this translates into a higher yield of VMs.
ViPSCs exhibit a distinct transcriptional and epigenetic signature

To gain insight into the molecular mechanisms by which ViPSCs preferentially differentiate into VMs, we performed genome-wide gene expression profiling and DNA methylation analyses. We harvested total RNA from three ViPSC lines and TiPSC lines, as well as an ESC line and parental VMs and TTFs for Affymetrix microarray analysis. Unsupervised hierarchical clustering based on transcriptome profiling revealed that ViPSC lines clustered separately from TiPSCs and ESCs, while overall the pluripotent stem cell lines and the parental somatic cell types grouped separately (Figure 5A). We also examined the global pattern of DNA methylation at single nucleotide resolution in the cells using reduced representation bisulfite sequencing (RRBS) [29]. Again, unsupervised hierarchical clustering based on global DNA methylation analysis showed that ViPSCs clustered separately from TiPSCs and ESCs, while overall the pluripotent stem cell lines and the parental somatic cell types clustered separately (Figure 5B).

Next we analyzed the transcriptional and epigenetic profiles of ViPSCs for genes that may be playing a role
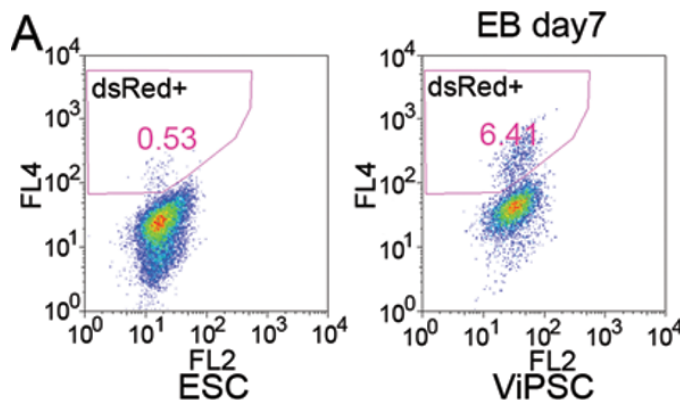

Nkx2.5

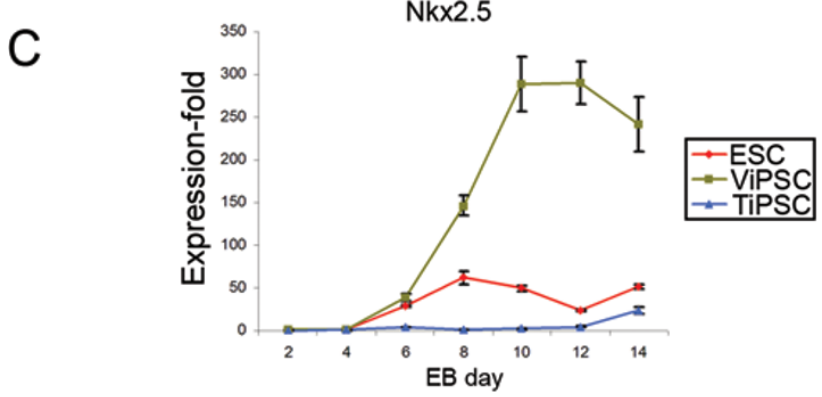

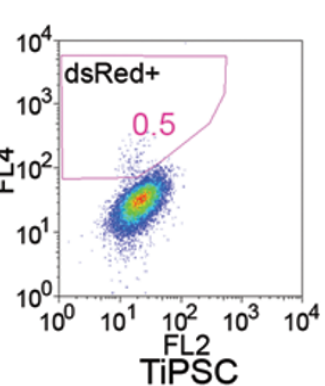

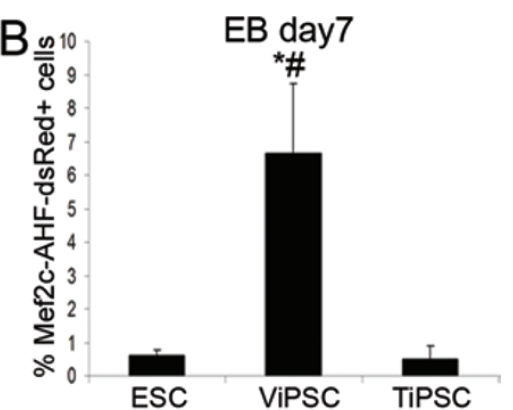

Is|1

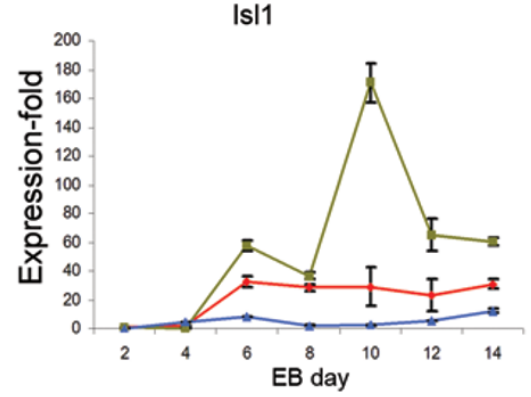

Figure 4 Early stage EBs from ViPSCs display a marked increase in cardiac progenitors. (A) Representative FACS dotplots of day 7 EBs showing increased numbers of Mef2c-AHF-dsRed ${ }^{+}$progenitor cells in ViPSCs. (B) Bar graph displaying percentages of $\mathrm{dsRed}^{+}$cells derived from early stage EBs from ViPSCs, TiPSCs, and ESCs. Asterisk denotes significantly different from ESC lines ( $n=6$ lines), $P<0.05$; pound sign denotes significantly different from TiPSC lines $(n=6$ lines), $P<0.05$. $n=6$ lines for ViPSCs. Bars represent standard error. (C) Quantitative RT-PCR demonstrating gene expression of Nkx2.5 and Islet-1 (Is/1) in EBs derived from ESCs, ViPSCs, and TiPSCs. RNAs from three lines of each cell type were pooled for analysis. The level of $N k x 2.5$ or Is/1 was normalized to that of $\beta$-actin and the level in day 2 EBs of ESC lines was set as one for relative comparison. Bars represent standard error. 
in ventricular myogenesis from pluripotent stem cells. An analysis of the genes that are more than 5-fold higher expressed in VMs relative to TTFs and the gene promot- ers that are hypomethylated in ViPSCs relative to TiPSCs demonstrated an overlap that is significantly higher than that expected by chance $(P=1.33 \mathrm{e}-4$, Fisher's exact test;
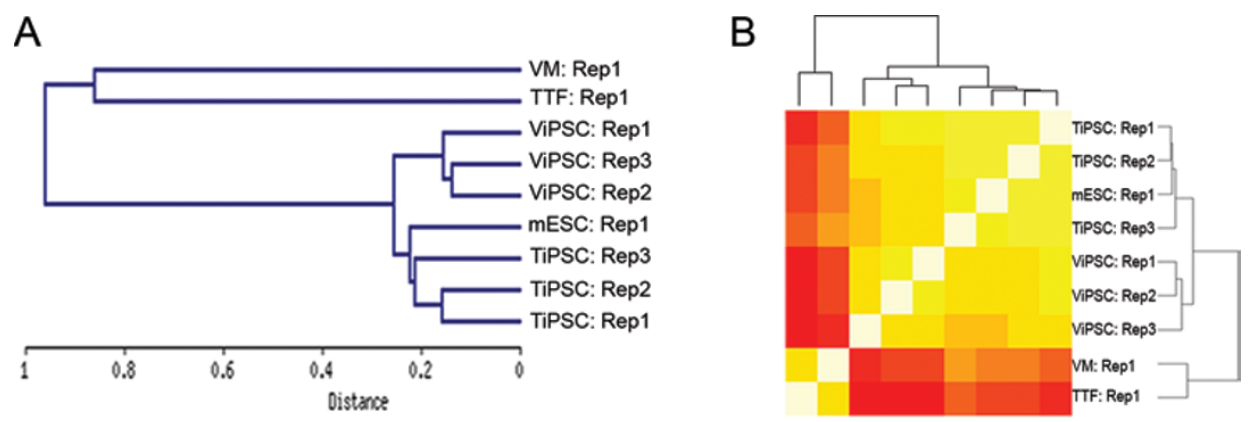

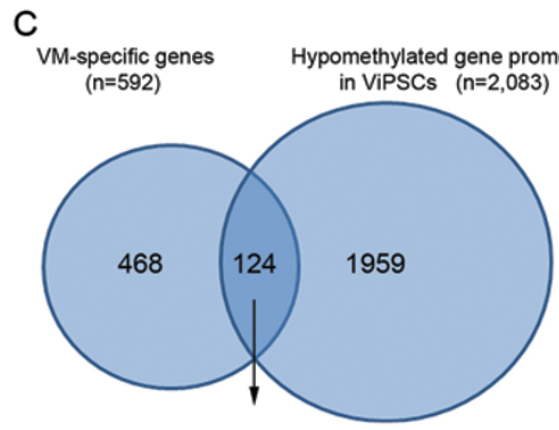

$P=1.33 \mathrm{e}-04$ (Fisher's exact test)

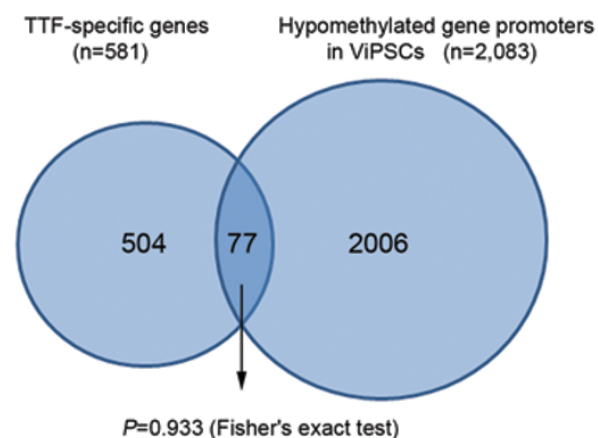

$P=0.933$ (Fisher's exact test)

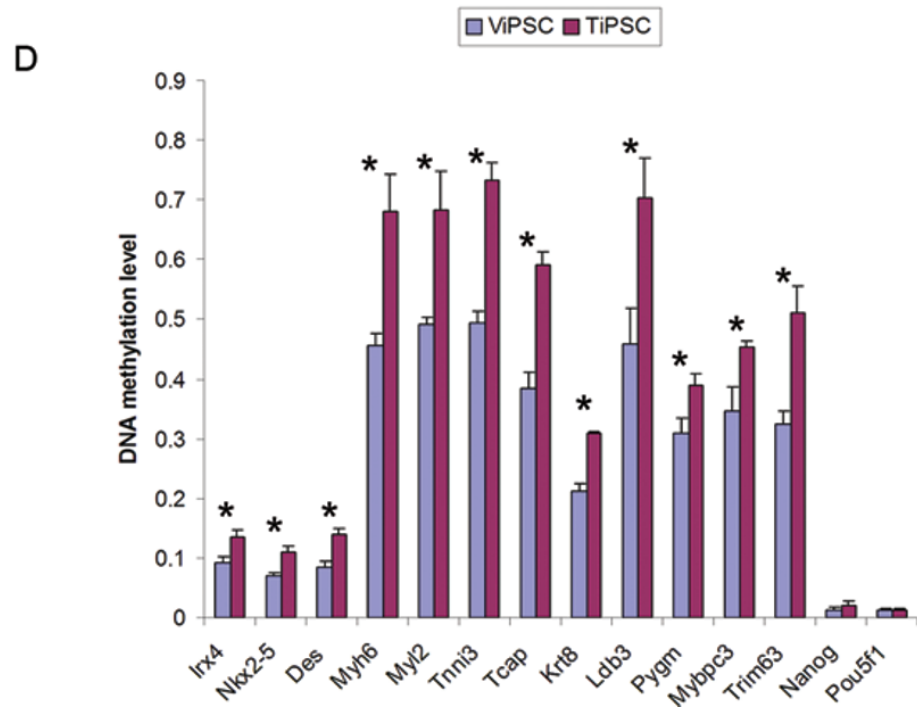

Figure 5 Transcriptional and epigenetic analysis of ViPSCs reveals a ventricular myogenic program. (A) Unsupervised clustering of the cell lines (passage 8 for pluripotent stem cells) based on genome-wide transcriptional profiling. (B) Unsupervised clustering of the cell lines (passage 8 for pluripotent stem cells) based on genome-wide DNA methylation levels analyzed by reduced representation bisulfite sequencing (RRBS). Red, low similarity; yellow, high similarity. (C) Venn diagrams of overlapping between genes with DNA hypomethylated promoters in ViPSCs (relative to TiPSCs) and genes highly expressed in VM or TTF. The Fisher's exact test $P$ values for overlapping between gene sets are indicated. (D) Bar graph showing DNA methylation levels in ViPSCs and TiPSCs at the promoter regions of cardiac genes and pluripotency genes. Error bars denote standard error. 
Figure 5C). By contrast, the overlap between genes overexpressed in TTFs and hypomethylated ViPSCs gene promoters was not significant $(P=0.933)$. These results suggest that ViPSCs retained an epigenetic signature that facilitates the activation of VM-specific genes. We narrowed the candidate ventricular myogenic genes to 87 genes by identifying the genes whose promoters also had a lower DNA methylation level in VMs relative to TTFs in order to focus on genes whose epigenetic signature was retained from VMs to the ViPSC state (Supplementary information, Table S1). This gene list includes the transcription factors $\operatorname{Ir} x 4$ and $N k x 2.5$. Irx 4 is an Iroquois homeobox transcription factor family that has been reported to activate VM-specific gene expression while suppressing atrial genes [30]. These genes also included some that encode for contractile proteins such as myosin heavy chain 6 (Myh6), myosin light chain 2v (Myl2), cardiac troponin I (Tnni3), and desmin (Des) (Figure 5D and Supplementary information, Table S1). These results demonstrate that a genome-wide transcriptional and epigenetic analysis of ViPSCs can help elucidate genes that play a critical role in the specification of VMs.

Memory in ViPSCs is relatively stable after extensive passaging

It has been reported that the iPSCs from some somatic cell types, including B cell, T cell, skeletal muscle pro- genitor, and granulocyte, essentially lose their memory after 16 passages [15], while the iPSCs from other somatic cell types, such as retinal-pigmented epithelial cells [31] appear to retain their memory despite extensive passaging. These results suggest that the origin of the iPSCs may affect the stability of the memory retained by iPSCs. In order to examine the effects of higher passage number on the cardiomyogenic potential in ViPSCs, we cultured them under identical conditions out to high passage numbers and performed FACS analysis using intracellular cTnT staining. At passage 16, the percentage of $\mathrm{cTnT}^{+}$cells from ViPSCs declined while the percentage of $\mathrm{cTnT}^{+}$from TiPSCs and ESC increased slightly; however, even at passage 24, ViPSCs yielded a discernibly higher percentage of cardiomyocytes than either TiPSCs or ESCs (Figure 6A), which suggests that the memory in ViPSCs is relatively stable.

\section{Discussion}

Our findings represent the first report of the generation and characterization of iPSCs from VMs and demonstrate that cardiomyocyte "memory" in iPSCs extends to individual cardiomyocyte subtypes and may be advantageously used to generate large numbers of beating VMs (Figure 6B). It remains unclear whether iPSC memory arises primarily from retained DNA methylation marks,
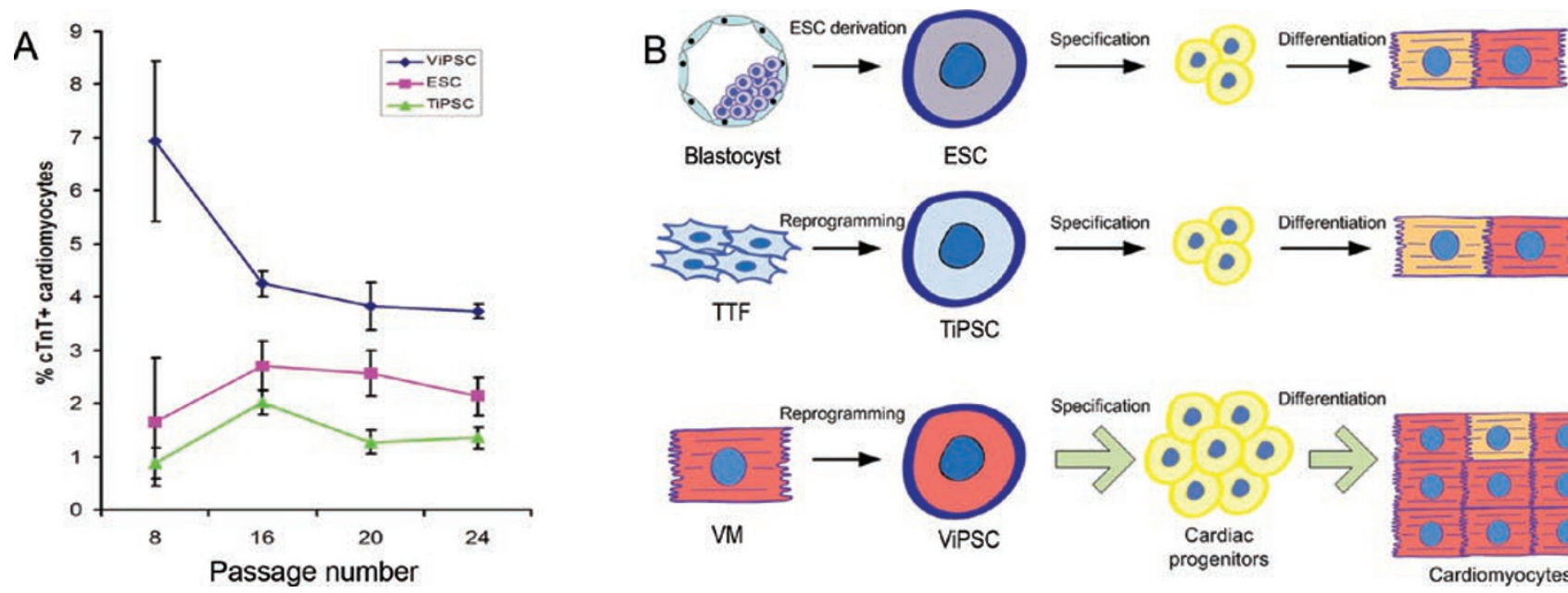

Figure 6 Effect of extensive passaging on the cardiomyogenic potential and the model depicting a higher ventricular myogenic potential of ViPSCs than ESCs and TiPSCs. (A) Line chart showing the change in the percentages of cTnT+ cardiomyocytes, as revealed by FACS analysis, along passage numbers after EB differentiation and cultivation of whole EBs in vitro. $P<0.05$ (significantly different) between ViPSC lines and ESC or TiPSC lines at all passage numbers, $n=6$ lines for each cell type, $t$-test. Error bars represent standard error. (B) Model depicting a higher ventricular myogenic potential of ViPSCs than ESCs and TiPSCs. The higher ventricular myogenic potential is demonstrated by more cardiomyocytes generated from ViPSCs and a vast majority of cardiomyocytes as ventricular myocytes (red) instead of atrial myocytes (yellow). The higher ventricular myogenic potential is achieved by increased numbers of cardiac progenitors. 
histone modifications, or a combination of both $[12,14$, $15]$. We speculate that the specific epigenetic mechanisms involved as well as the stability of epigenetic "memory" to repeated passaging may vary with each somatic cell type.

Analysis of ViPSCs and their parental VMs revealed genes that may play a role in the specification of cardiovascular progenitors and VMs. These genes include transcription factor genes as well as genes that encode contractile proteins and are likely part of the "late cardiomyogenic program". Interestingly, pair-wise comparisons revealed that all ViPSCs possessed a trend of global DNA hypomethylation at sites examined by RRBS (mostly CpG-rich gene promoters) when compared to TiPSCs and a reference ESC line, which was not observed in parental VMs (data not shown). This phenomenon may reflect a transient state in the reprogramming of cardiomyocytes to iPSCs [14]. Alternatively, the mild DNA hypomethylation in ViPSCs may contribute to ventriculogenesis by rendering the epigenome in a more "permissive" state for myogenic gene expression. This hypothesis is consistent with mechanism of action of the DNA demethylating agent, 5-azacytidine, which has been used to induce cardiomyogenic gene expression in Sca- $1^{+}$cardiovascular progenitors [32] and human ESCs [26]. Moreover, DNA demethylation has been implicated in the epigenetic regulation of skeletal muscle specification [33, 34]. While a number of significant challenges remain, the findings here suggest that the reprogramming of human VMs to human ViPSCs may be a tractable strategy of deriving large numbers of VMs for cardiac regenerative medicine.

It will be interesting to test whether the iPSCs derived from human VMs also display an increased ventricular myogenic potential as their mouse counterpart. While reprogramming of primary human VMs to iPSCs is technically challenging, a conditional reprogramming strategy similar to the one used in this manuscript can be employed to generate secondary iPSCs from human ESC- or iPSC-derived VMs. Comparison between human iPSCs from VMs and other parental cell types may provide useful information concerning the epigenetic or genetic regulation on ventricular myogenesis from human pluripotent stem cells. Such information may eventually facilitate the development of methods to derive VMs from pluripotent stem cells in an efficient manner.

\section{Materials and Methods}

Mouse lines and constructs

The Mlc $2 v^{\text {Crel+ }} ;$ Rosa $26^{\text {floxYFP/rtA }}$; TgMef $2 c-A H F-d s R e d$ compound transgenic animals were obtained by crossing $M l c 2 v^{\text {Cre/ }}$; Rosa $26^{\text {rtTA rtTA }}$ males with Rosa $26^{\text {floxFPflloxYFP }} ; \operatorname{TgMef} 2 c-A H F-d s R e d$ females. Mlc $2 v^{\mathrm{Cre} / \mathrm{+}}$ knock-in mouse line was generated in the lab as described, and $M l c 2 v^{C r e}$ is expressed specifically in VMs [18]. Rosa $26^{\text {floxYFPIfloxYFP }}$ conditional reporter mouse line [19] was purchased from the Jackson Laboratory. Rosa2 $6^{r t T A \text { rtTA }}$ mouse line was kindly provided by Dr Konrad Hochedlinger [20]. The TgMef2c$A H F$-dsRed transgenic mouse line was generated in the lab as described [22]. The constructs used to produce lentivirus for iPSC derivation were kindly provided by Dr Konrad Hochedlinger [16].

\section{Production of lentivirus}

To produce infectious lentiviral particles, 293FT cells (Invitrogen) were cultured on $10-\mathrm{cm}$ dishes and transfected with the expression vectors together with the packaging plasmids pMD2G and pCMV-dR8.74 with Fugene HD (Roche). The viral particles for each reprogramming factor were produced individually. Viruscontaining medium was harvested on 2 consecutive days $48 \mathrm{~h}$ after transfection and used for infection without concentration.

\section{Generation of the primary iPSCs from cardiac fibroblasts}

To collect cardiac fibroblasts from neonatal pups, hearts were digested with trypsin (USB) at $4{ }^{\circ} \mathrm{C}$ overnight, followed by collagenase type 2 (Worthington) at $37^{\circ} \mathrm{C}$ for $30 \mathrm{~min}$, and dissociated by triturating $\sim 10$ times. Cardiac fibroblasts were enriched by differential centrifugation and plating. In all, 20000 cardiac fibroblasts were plated per well on a six-well plate the day before infection. Virus-containing medium was applied on the cells with polybrene (Sigma). Two infections were performed on the cells. At $48 \mathrm{~h}$ after infection, the medium was changed to ESC medium with $1 \mu \mathrm{g} / \mathrm{ml}$ doxycycline (Sigma), which was withdrawn in 12 days. The putative iPSCs colonies were picked 2 days later after the withdrawal of doxycycline.

Generation of the secondary iPSCs from ventricular cardiomyocytes and TTFs

The ventricular cardiomyocytes and TTFs from one neonatal chimeric pup were used for the generation of the secondary iPSCs. To collect cardiomyoctes, the heart was digested by trypsin (USB) at $4{ }^{\circ} \mathrm{C}$ overnight, followed by collagenase type 2 (Worthington) at $37^{\circ} \mathrm{C}$ for $30 \mathrm{~min}$, and dissociated by triturating 10 times. The cardiomyocyte fraction was enriched by differential centrifugation and plating, after which the cardiomyocytes were plated directly on MEF feeder for the derivation of the secondary iPSCs. To collect TTFs, the tail tip was minced in trypsin and cultured with DMEM with $10 \%$ FBS for 4 days. The attached TTFs were detached with trypsin and plated on MEF feeder for the derivation of the secondary iPSCs. After the cardiomyocytes and TTFs were plated on MEF feeder, doxycycline was added into the medium to re-induce the expression of the reprogramming transgenes. In 2 weeks, putative iPSC colonies emerged. For the secondary iPSCs from ventricular cardiomyocytes, $\mathrm{YFP}^{+}$colonies were picked for further study. For the secondary iPSCs from TTFs, colonies were picked randomly.

Establishment of Mlc2 $v^{\text {Cre/+ }} ;$ Rosa26 $6^{\text {floxYFP/rtTA }} ; \operatorname{TgMef2c}$ AHF-dsRed compound transgenic ESC lines

Timed matings were performed between $M l c 2 v^{\text {Cre/t }} ; \operatorname{Rosa} 26^{\text {rtTA/rtTA }}$ males with Rosa $26^{\text {YFP/YFP }} ; \mathrm{TgMef} 2 \mathrm{c}$-AHF-dsRed females. On day 3.5 PC, females were sacrificed and the blastocysts flushed from the uterine horns with M2 medium (Sigma-Aldrich). The zona 
pellucida was removed with acidic Tyrode's Solution (SigmaAldrich) and the blastocysts were further washed three times with M2 media. The blastocysts were then plated onto MEF feeder cells with ESC derivation medium (DMEM with 15\% KOSR, penicillin/streptomycin, sodium pyruvate, nonessential amino acids (NEAA), and leukemia inhibitory factor (LIF) (Chemicon, CA, USA)).

\section{Karyotyping analysis}

Karyotyping analysis was performed based on a published protocol [35]. Briefly, iPSCs were treated with $0.02 \mu \mathrm{g} / \mathrm{ml}$ Colcemid (Sigma) for 45-60 min and dissociated with trypsin. Trypsinization was stopped by PBS containing 10\% FBS. The dissociated cells were washed with PBS once and incubated with hypotonic solution $\left(0.56 \% \mathrm{KCl}\right.$ in $\left.\mathrm{H}_{2} \mathrm{O}\right)$ at room temperature for $10 \mathrm{~min}$. Freshly made Carnoy's fixative (1:3 glacial acetic acid:methanol, v/v) was used to fix the swollen cells for two cycles. Cells in the fixative were dropped on cold glass slides and analyzed under optical microscope. At least 20 cells were counted per line.

\section{Chimera assay}

Individual iPSCs/ESCs were microinjected into E3.5 blastocysts from $\mathrm{C} 57 \mathrm{Bl} / 6$ females and implanted into the uterus of pseudopregnant CD-1 foster mothers. ViPSCs had been YFP upon their derivation and were readily visible under fluorescent microscope. To trace the ESCs and TiPSCs, the conditional reporter allele Rosa $26^{f l o x Y F P}$ was activated by transient expression of an exogenous Cre in the cells before injection. The exogenous Cre was expressed under the control of CMV promoter from a circular DNA plasmid that was introduced into the cells through electroporation. YFP-positive ESCs or TiPSCs were purified through FACS, expanded and subjected to blastocyst injection. The chimeras were categorized based on the contribution of iPSCs or ESCs: type 1 chimeras demonstrated $\mathrm{a} \geq 50 \%$ contribution to the embryo overall including the heart; type 2 chimeras demonstrated a $<50 \%$ contribution overall but a much higher contribution to the heart; and type 3 embryos demonstrated a $<50 \%$ contribution overall with an equal or lesser contribution to the heart.

\section{Alkaline phosphatase activity assay}

iPSCs were cultured in a medium containing LIF before the assay. On the day of the assay, the medium was removed and the cells were processed with the reagents from the alkaline phosphatase substrate kit (Vector Laboratories).

\section{In vivo teratoma formation}

ESCs or iPSCs were allowed to differentiate into EB by growing in suspension culture in the absence of recombinant LIF at $37^{\circ} \mathrm{C}$ for 7 days. Two to four EBs were grafted under the kidney capsule of SCID/NOD mice, and groups of mice were sacrificed for analysis 4 weeks after transplantation. Graft acceptance was defined as an increase in diameter to $>5 \mathrm{~mm}$, vascularization, and the lack of leukocyte infiltration and tissue damage, revealed by histology. The readout of transplantation was restricted by 28 days to minimize tumor-associated risks.

In vitro differentiation of $i P S C s / E S C s$ through EB formation

ESCs were cultured for at least two passages and then adapted for EB formation in adaptation medium (IMDM (Invitrogen) with
$15 \%$ FBS, penicillin/streptomycin, sodium pyruvate, NEAA, and LIF) for 2 days. The adapted cells were digested to individual cells and re-suspended in differentiation medium (IMDM with $15 \% \mathrm{FBS}$, penicillin/streptomycin, NEAA, and ascorbic acid) at a concentration of 45000 cells $/ \mathrm{ml}$. Hanging drops containing about 540000 cells were made with the cell suspension and cultured for 2 days, during which the cells in each hanging drop aggregated to form one EB. EBs were cultured in differentiation medium until the day of analysis.

To enhance the growth of cardiomyocytes, EBs were dissociated with trypsin (Invitrogen) on day 7, and the individual cells were cultured on gelatin with a medium specialized for cardiomyocytes (DMEM/F-12 (Invitrogen), 20\% FCS, 5\% horse serum, $2 \mathrm{mM}$ L-glutamine, $0.1 \mathrm{M}$ NEAA, $3 \mathrm{mM}$ sodium pyruvate, and $1 \mu \mathrm{g} / \mathrm{ml}$ bovine insulin).

\section{Transcriptional profiling and quantitative RT-PCR}

RNA from ESCs/iPSCs, primary VMs, TTFs, or EBs was extracted with RNeasy mini kit (Qiagen). For transcriptional profiling, RNA was converted to complementary RNA (cRNA) and hybridized to the Affymetrix mouse genome 4302.0 chip. Hybridization results were analyzed with bioinformatics tools available at the website of National Institute of Aging (NIA). The raw data of transcriptional profiling has been deposited to Gene Expression Omnibus (www.ncbi.nlm.nih.gov/geo), and the accession number is GSE32598. For quantitative RT-PCR, cDNA was synthesized with iScript kit (Bio-Rad). Real-time PCR was performed using the I-cycler system with SYBR Green substrate (BioRad) for 40 cycles. Primer sequences are available on request.

\section{Immunofluorescence studies}

Antibodies used in this study include antibody against the following proteins: SSEA1 (Hybridoma Bank at Iowa), Nanog (Cosmo Bio), Oct3/4 (Santa Cruz), cardiac Troponin T (NeoMarkers), cardiac Troponin I (Abcam), and Mlc2v (F109.3E1, Biocytex). The cells were fixed with $4 \%$ paraformaldehyde (PFA) and blocked with PBS containing 10\% FBS. Then the cells were incubated with blocking solution with the primary antibody. Subsequently, samples were incubated with the appropriate secondary antibodies conjugated with Alexa-Fluoro 488 or 594 (Molecular Probes/Invitrogen).

\section{Intracellular FACS}

EBs were dissociated with trypsin followed by collagenase A and B. The cells were then fixed with 2\% PFA (Sigma), blocked and permeabilized with PBS containing $10 \%$ FBS and $0.5 \%$ Tween 20. Cells were then incubated with the primary antibody overnight at $4{ }^{\circ} \mathrm{C}$, washed with the blocking solution, and incubated with the secondary antibody for $1 \mathrm{~h}$ at room temperature. They were washed with PBS and analyzed with FACSCalibur (BD Biosciences). Antibodies used for intracellular FACS analysis include those against cardiac Troponin T (NeoMarkers) and dsRed (Clontech). Secondary antibodies conjugated with Alexa-660, Alexa-647, or APC (Molecular Probes/Invitrogen) were used.

To collect the cells for RNA extraction, EBs were dissociated as above. The individual cells were fixed with DEPC-treated $75 \%$ ethanol, washed with RNase-free PBS, and incubated with the primary antibody against cTnT (NeoMarkers) in PBS containing $1 \%$ acetylated BSA (B2518, Sigma). Subsequently, samples were 
incubated with the secondary antibody conjugated with Alexa-647 or Alexa660. They were washed and $\mathrm{cTnT}^{+}$cells were collected with FACSAria (BD Biosciences).

\section{Electrophysiology}

ESCs/iPSCs were differentiated through EB formation and beating areas in day 14-16 EBs were manually dissected. Cells were dissociated with collagenase II (Worthington Biological) and plated on fibronectin-coated coverslips. Patch electrodes were filled with an intracellular solution containing $140 \mathrm{mM}$ potassium gluconate, $10 \mathrm{mM} \mathrm{NaCl}, 2 \mathrm{mM} \mathrm{MgCl}$, $10 \mathrm{mM}$ HEPES, $1 \mathrm{mM}$

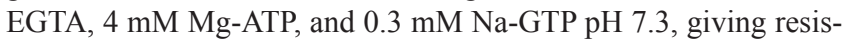
tances of 2-5 M $\Omega$. Spontaneous cardiomyocyte action potentials were recorded at room temperature, in culture medium, using the whole-cell patch clamp method in current clamp mode with an Axopatch 200A amplifier (Axon Instruments/Molecular Devices, Sunnyvale, CA, USA).

\section{Reduced representation bisulfite sequencing (RRBS)}

Genomic DNA was isolated from the cells using Purolink Genomic DNA Mini Kit (Invitrogen). RRBS was performed and the sequencing results were analyzed as previously described [36]. Briefly, genomic DNA was digested with methylation-insensitive restriction enzyme MspI and ligated to methylated Illumina adapter. Gel purification was employed to select for the fragments of 40$220 \mathrm{bp}$, which were subjected to bisulfite conversion. The converted DNA was PCR amplified and used to construct libraries for sequencing. The raw sequencing reads were analyzed and aligned to a mouse genome sequence that had been MspI-digested and size-selected in silico using Maq's bisulfite alignment mode [37]. DNA methylation calling was performed using custom software [38]. Next, we calculated mean DNA methylation levels for all gene promoters that were covered by a minimal number of DNA methylation measurements [36]. Data processing was performed by custom Python (http://python.org/) and R (http://www.r-project. org/) scripts. All data are publicly available for visual browsing and download at http://vipsc-memory.computational-epigenetics.org.

\section{Statistical analysis}

$t$-test was used for the comparisons between ESCs, ViPSCs, and TiPSCs unless otherwise indicated. The analysis was performed with Microsoft Excel and the difference was considered statistically significant when $P<0.05$. The $\chi^{2}$-test was used to compare the distribution of the various chimera types between the chimeras from ESCs, ViPSCs, and TiPSCs. The difference was considered statistically significant when $P<0.05$. Fisher's exact test was used to assess the overlapping between the genes specifically expressed in VMs and the hypomethylated gene promoters in ViPSCs. We compared gene expression profiles in VM and TTF (measured by Affymetrix mouse 4302.0 arrays), and genes with $>$ 5-fold enrichment of expression in VM relative to TTF were included as "VMspecific genes". The promoters with a significantly lower DNA methylation level in ViPSCs relative to TiPSCs ( $P$ Value ( $t$-test) $<5.0 \mathrm{e}-03, n=3$ and a minimum RRBS coverage $>5$ reads) are called as "hypomethylated gene promoters in ViPSCs", the list of which is used to generate Venn diagrams in Figure 4C. The $\chi^{2}$ and Fisher's exact tests were performed with R (http://www.r-project. org/).

\section{Acknowledgments}

We would like to thank Laura Prickett-Rice, Kat Folz-Donahue, and Sutanuka Lahiri of the Harvard Stem Cell Institute Flow Cytometry Core Facility for assistance with FACS analysis, Chuang Du of the Tufts Electrophysiology Core for assistance with electrophysiology recordings, Jie Zhao and members of the Wellman Photopathology Core for assistance with histology, Patricia Follett for assistance with blastocyst injections, and Elizabeth Bearrick for assistance with experiments. We would like to thank Konrad Hochedlinger (Massachusetts General Hospital, Harvard University, USA) for sharing the Rosa2 $6^{r \text { tTA/rtTA }}$ mouse line as well as the lentiviral constructs for iPSC derivation. We would like to thank Lei Bu, Emil Hansson, members of the Chien laboratory, and Konrad Hochedlinger for helpful discussion. HX is supported by Massachusetts General Hospital Executive Committee of Research Fund for Medical Discovery. BAY has received support under NIH Training Grant (5T32HL007208-32) and the MGH ECOR Fund for Medical Discovery. HW is supported by a Jane Coffin Childs postdoctoral fellowship. KOL holds a Croucher Foundation Fellowship. This work is partially funded by GlaxoSmithKline Pharmaceuticals.

\section{References}

1 Yoshida Y, Yamanaka S. Recent stem cell advances: induced pluripotent stem cells for disease modeling and stem cellbased regeneration. Circulation 2010; 122:80-87.

2 Efe JA, Hilcove S, Kim J, et al. Conversion of mouse fibroblasts into cardiomyocytes using a direct reprogramming strategy. Nat Cell Biol 2011; 13:215-222.

3 Ieda M, Fu JD, Delgado-Olguin P, et al. Direct reprogramming of fibroblasts into functional cardiomyocytes by defined factors. Cell 2010; 142:375-386.

4 Laflamme MA, Murry CE. Regenerating the heart. Nat Biotechnol 2005; 23:845-856.

5 Mauritz C, Schwanke K, Reppel M, et al. Generation of functional murine cardiac myocytes from induced pluripotent stem cells. Circulation 2008; 118:507-517.

6 Narazaki G, Uosaki H, Teranishi M, et al. Directed and systematic differentiation of cardiovascular cells from mouse induced pluripotent stem cells. Circulation 2008; 118:498-506.

7 Epstein JA. Franklin H. Epstein Lecture. Cardiac development and implications for heart disease. N Engl J Med 2010; 363:1638-1647.

8 Ng SY, Wong CK, Tsang SY. Differential gene expressions in atrial and ventricular myocytes: insights into the road of applying embryonic stem cell-derived cardiomyocytes for future therapies. American J Physiol Cell Physiol 2010; 299:12341249.

9 He JQ, Ma Y, Lee Y, Thomson JA, Kamp TJ. Human embryonic stem cells develop into multiple types of cardiac myocytes: action potential characterization. Circ Res 2003; 93:3239.

10 Moore JC, Fu J, Chan YC, et al. Distinct cardiogenic preferences of two human embryonic stem cell (hESC) lines are imprinted in their proteomes in the pluripotent state. Biochem Biophys Res Commun 2008; 372:553-558. 
11 Bar-Nur O, Russ HA, Efrat S, Benvenisty N. Epigenetic memory and preferential lineage-specific differentiation in induced pluripotent stem cells derived from human pancreatic islet Beta cells. Cell Stem Cell 2011; 9:17-23.

12 Kim K, Doi A, Wen B, et al. Epigenetic memory in induced pluripotent stem cells. Nature 2010; 467:285-290.

13 Lister R, Pelizzola M, Kida YS, et al. Hotspots of aberrant epigenomic reprogramming in human induced pluripotent stem cells. Nature 2011; 471:68-73.

14 Ohi Y, Qin H, Hong C, et al. Incomplete DNA methylation underlies a transcriptional memory of somatic cells in human iPS cells. Nat Cell Biol 2011; 13:541-549.

15 Polo JM, Liu S, Figueroa ME, et al. Cell type of origin influences the molecular and functional properties of mouse induced pluripotent stem cells. Nat Biotechnol 2010; 28:848855.

16 Stadtfeld M, Maherali N, Breault DT, Hochedlinger K. Defining molecular cornerstones during fibroblast to iPS cell reprogramming in mouse. Cell Stem Cell 2008; 2:230-240.

17 Wernig M, Lengner CJ, Hanna J, et al. A drug-inducible transgenic system for direct reprogramming of multiple somatic cell types. Nat Biotechnol 2008; 26:916-924.

18 Chen J, Kubalak SW, Minamisawa S, et al. Selective requirement of myosin light chain $2 \mathrm{v}$ in embryonic heart function. $J$ Biol Chem 1998; 273:1252-1256.

19 Srinivas S, Watanabe T, Lin CS, et al. Cre reporter strains produced by targeted insertion of EYFP and ECFP into the ROSA26 locus. BMC Dev Biol 2001; 1:4.

20 Hochedlinger K, Yamada Y, Beard C, Jaenisch R. Ectopic expression of Oct-4 blocks progenitor-cell differentiation and causes dysplasia in epithelial tissues. Cell 2005; 121:465-477.

21 Qyang Y, Martin-Puig S, Chiravuri M, et al. The renewal and differentiation of $\mathrm{Isll}^{+}$cardiovascular progenitors are controlled by a Wnt/beta-catenin pathway. Cell Stem Cell 2007; 1:165-179.

22 Domian IJ, Chiravuri M, van der Meer P, et al. Generation of functional ventricular heart muscle from mouse ventricular progenitor cells. Science 2009; 326:426-429.

23 Laflamme MA, Chen KY, Naumova AV, et al. Cardiomyocytes derived from human embryonic stem cells in pro-survival factors enhance function of infarcted rat hearts. Nat Biotechnol 2007; 25:1015-1024.

24 Fijnvandraat AC, Lekanne Deprez RH, Moorman AF. Development of heart muscle-cell diversity: a help or a hindrance for phenotyping embryonic stem cell-derived cardiomyocytes. Cardiovasc Res 2003; 58:303-312.
25 Fu JD, Jiang P, Rushing S, et al. $\mathrm{Na}^{+} / \mathrm{Ca}^{2+}$ exchanger is a determinant of excitation-contraction coupling in human embryonic stem cell-derived ventricular cardiomyocytes. Stem Cells Dev 2010; 19:773-782.

26 Mummery C, Ward-van Oostwaard D, Doevendans P, et al. Differentiation of human embryonic stem cells to cardiomyocytes: role of coculture with visceral endoderm-like cells. Circulation 2003; 107:2733-2740.

27 Laugwitz KL, Moretti A, Caron L, Nakano A, Chien KR. Islet1 cardiovascular progenitors: a single source for heart lineages? Development 2008; 135:193-205.

28 Wu SM, Fujiwara Y, Cibulsky SM, et al. Developmental origin of a bipotential myocardial and smooth muscle cell precursor in the mammalian heart. Cell 2006; 127:1137-1150.

29 Meissner A, Mikkelsen TS, Gu H, et al. Genome-scale DNA methylation maps of pluripotent and differentiated cells. $\mathrm{Na}$ ture 2008; 454:766-770

30 Bao ZZ, Bruneau BG, Seidman JG, Seidman CE, Cepko CL. Regulation of chamber-specific gene expression in the developing heart by Irx4. Science 1999; 283:1161-1164.

$31 \mathrm{Hu}$ Q, Friedrich AM, Johnson LV, Clegg DO. Memory in induced pluripotent stem cells: reprogrammed human retinalpigmented epithelial cells show tendency for spontaneous redifferentiation. Stem Cells 2010; 28:1981-1991.

32 Oh H, Bradfute SB, Gallardo TD, et al. Cardiac progenitor cells from adult myocardium: homing, differentiation, and fusion after infarction. Proc Natl Acad Sci USA 2003; 100:12313-12318.

33 Chiu CP, Blau HM. 5-Azacytidine permits gene activation in a previously noninducible cell type. Cell 1985; 40:417-424.

34 Palacios D, Puri PL. The epigenetic network regulating muscle development and regeneration. J Cell Physiol 2006; 207:1-11.

35 Nagy A, Gertsenstein M, Vintersten K, Behringer R. Detection and analysis of mouse genome alterations and specific sequences. Manipulating the mouse embryo (3rd edition). Cold Spring Harbor Laboratory Press: New York 2003:534-539.

36 Bock C, Kiskinis E, Verstappen G, et al. Reference maps of human ES and iPS cell variation enable high-throughput characterization of pluripotent cell lines. Cell 2011; 144:439-452.

37 Li H, Ruan J, Durbin R. Mapping short DNA sequencing reads and calling variants using mapping quality scores. Genome Res 2008; 18:1851-1858.

$38 \mathrm{Gu} \mathrm{H}$, Bock C, Mikkelsen TS, et al. Genome-scale DNA methylation mapping of clinical samples at single-nucleotide resolution. Nat Methods 2010; 7:133-136.

(Supplementary information is linked to the online version of the paper on the Cell Research website.) 